\title{
Bone Markers in the Treatment of Cancer Related Bone Disease in Patients with Metastatic Breast Cancer
}

\author{
Brančíková D*, Mechl Z, Adam Z, Protivánková M, Ostřížková L, Bednařík O and Mayer J
}

Department of Internal Medicine, Hematology and Oncology, University Hospital Brno and Masaryk University, School of Medicine, Czech Republic

\begin{abstract}
Bone metastases and treatment-induced osteoporosis are frequently the maincauses of morbidity in patients with malignancies. Monitoring the level of bone markers (markers of bone metabolism) has been fairly well mapped in osteoporosis, where medical procedures can be modified according to the kinetics of marker levels before an answer can be evaluated by densitometry and before the onset of fractures. In bone metastases the role of these levels is not clear. The metabolic markers of bone resorption under review in this set were ß-Cross Laps (CTX) - a peptide that is a part of $\mathrm{C}$ - telopeptide, and $\mathrm{N}$-terminal propeptide of collagen type 1 (P1NP).

Material and methods: We monitored a group of 52 female patients with metastatic breastcancer. The patients received appropriate systemic treatment based on the immunohistochemistry of the tumor; the treatment consisted of hormone therapy or chemotherapy, and included parenteral bisphosphonates (ibandronate and zoledronic acid alternately). Routine biochemical tests and blood count done prior to the initiation of therapy also included taking laboratory markers of bone metabolism CTX and P1NP and measuring bone mineral density (according to T-score). These bone markers were then checked in three, six, nine and twelve months, and matched with the progress of the disease. Total monitoring time was fifteen months.

Results: The patients in this set whose CTX value in the first sampling was less than 0.425 ran 8.5 times higher risk of death; the patients whose P1NP value reached more than 74 in the first collection ran 8.7 times higher risk of death. According to Cox proportional-hazards regression analysis for CTX, the significance level of $p$-value was 0.0452 and $\mathrm{HR}$ was 8.516 (95\% $\mathrm{Cl} 1.047$ to 69.262$)$, a difference which is not statistically significant. Regarding $\mathrm{P} 1 \mathrm{NP}$ in Cox regression analysis, the significance level of $\mathrm{p}$-value is 0.0433 and $\mathrm{HR} 8.673(95 \% \mathrm{Cl} 1.067$ to 70.520). Even this difference is therefore not statistically significant. When comparing the kinetics of marker levels, the difference is below statistical significance: $p$-value 0.6131 for P1NP and p-value 0.6357 for CTX.
\end{abstract}

Conclusion: The results of this study confirm a correlation between the starting levels of CTX and P1NP with the overall survival rate, which corresponds to the other results presented in literature.

\section{Introduction}

Bone metastases and treatment-induced osteoporosis are frequently the main causes of morbidity in patients with malignancies. The main indicator of low treatment efficacy of bone metastases is the incidence of skeleton-related events (SRE). Monitoring the levels of bone markers (biochemical markers of bone metabolism) has been fairly well mapped in osteoporosis, where medical procedures can be modified in accordance with the kinetics of marker levels before the answer can be evaluated by densitometry and before the development of a fracture $[1,2]$. In bone metastases, the role of these levels is not clear $[3,4]$.

The first metabolic marker of bone resorption under review in this set was ß-Cross Laps (CTX) - a peptide that is a part of the C-telopeptide. It is localized on the beta chains of collagen type 1 and includes a position for possible pyridine coupling. It is easier to determine it from blood, something which is performed on an automated analyzer on a daily basis. It is recommended to determine CTX beta for monitoring the effectiveness of anti-resorptive therapy (e.g. by bisphosphonates, HRT) in osteoporosis or other bone disorders. In bone metastases, the optimal specificity seems to be $(90 \%)$ at the value of $0.4 \mathrm{~g} / \mathrm{l}$. The sensitivity of this methodology is around $70 \%$ in bone metastases with breast cancer and $67 \%$ in prostate cancer.

What is also currently recommended for monitoring bone formation is $\mathrm{N}$-terminal propeptide of collagen type 1 (P1NP), especially for osteoblastic metastases. P1NP is a highly specific marker showing the formation of osteoblastic (and also mixed) metastases; it shows little circadian variation, it can be detected even in serum and it has a relatively low intra-individual variation [5-8]. Should it be confirmed that patients with skeletal events show different kinetics in bone markers during treatment than patients without SRE, we would obtain another simple tool that would provide advance warning of an imminent risk of SRE, which is clearly a prognostic factor for shorter survival $[9,10]$.

\section{Material and Methods}

From March 2009 to December 2011, we studied a group of 52 female patients with metastatic breast cancer (3 patients with only bone metastases, others had two or more organs affected, but in

*Corresponding author: Brancikova Dagmar, Department of Internal Medicine Hematology and Oncology, Jihlavská 340/20, 62500 Brno Building L, Complex University, University Hospital Brno and Masaryk University, School of Medicine Hospital Brno-Bohunice, Bohunice, Brno, Czech Republic, Tel: +420 532232508 E-mail: dagmar.brancikova@fnbrno.cz

Received November 12, 2013; Accepted January 20, 2014; Published January 27, 2014

Citation: Brančíková D, Mechl Z, Adam Z, Protivánková M, Ostřǐ̌ková L, et al. (2014) Bone Markers in the Treatment of Cancer Related Bone Disease in Patients with Metastatic Breast Cancer. J Cancer Sci Ther 6: 027-031. doi:10.4172/19485956.1000244

Copyright: @ 2014 Brančíková D, et al. This is an open-access article distributed under the terms of the Creative Commons Attribution License, which permits unrestricted use, distribution, and reproduction in any medium, provided the original author and source are credited. 
each case one was a bone). The diagnosis of bone dissemination was determined both by means of bone scintigraphy, and confirmed by another imaging method - an X-ray or CT scan (3x NMR spine, $3 \mathrm{x}$ $\mathrm{CT} / \mathrm{PET})$. The patients received adequate systemic therapy based on the immunohistochemistry of the tumor, i.e., hormone therapy or chemotherapy, and also the parenteral bisphosphonate (alternately ibandronate and zoledronic acid). The routine biochemical tests and blood count done prior to the initiating therapy also included taking laboratory markers of bone metabolism CTX and P1NP and measuring the bone mineral density (according to the $\mathrm{T}$-score). These bone markers were then checked in three, six, nine and twelve months, and matched with the progress of the disease. The total monitoring time was fifteen months. During each examination, the use of analgesics was recorded, as well as the occurrence of sudden SRE or other complications; before the end of monitoring, densitometry was done again. See Table 1 for the Monitoring Flow Sheet. The evaluation deals separately with the two groups (with or without SRE), and compares the kinetics of the levels of both markers during treatment as well as the occurrence of adverse effects and complications during the course of treatment, and the number of deaths.

\section{The treatment set}

A group of 52 women at the age from 31 to 81 (the median was 63.5 years) with metastatic breast cancer and bone metastases. The monitoring period was from March 2009 to December 30, 2011. All patients were examined by scintigraphy, with a negative finding in 2 patients with clinical symptoms, where the finding was confirmed by CT examination of the site according to clinical symptoms.

\section{Treatment by bisphosphonates}

After the first examination, patients were alternately treated by zoledronic acid or ibandronate. Ibandronate was replaced by zoledronic acid only in five patients with reduced left ventricular ejection fraction and in the treatment of a light heart failure, due to the volume of hydration administered.

\section{Antineoplastic treatment}

In all cases there was first-line palliative treatment applied. It was administered according to the immunoprofile: the hormonal treatment was applied with a steroid receptor positivity of over $10 \%$ and negativity of HER 2 neu; in other cases, chemotherapy treatment was administered.

\section{Hormonal therapy}

It was administered in thirty patients using tamoxifen ( 5 patients) or thearomatase inhibitor (AI) in 19 patients, and selective destructor of steroid receptor (SERMD - fulvestrant) in one patient. In four patients, letrozole was replaced by anastrozole during the treatment, in order to achieve a better profile of side effects and better tolerability.

\section{Antineoplastic chemotherapy}

It was administered in 33 patients. In 4 patients there was apositive HER 2 neu receptor; in one, treatment started in taxane/ trastuzumab regimen, and in three patients there was the so-called triple-positive immunoprofile, so in the first line letrozole/herceptin was administered. 20 patients were triple-negative, of which one was treated by avastin+paclitaxel with a good result. Chemotherapy was administered according to the patient's general condition, laboratory values, left ventricular ejection fraction, previous treatment, effect and tolerability.

\begin{tabular}{|c|c|c|c|c|c|c|}
\hline & Day & Month & Month & Month & Month & Month \\
\hline & 0 & 3 & 6 & 9 & 12 & 15 \\
\hline CTX,P1NP, & $x$ & $\mathbf{x}$ & $\mathbf{x}$ & $\mathbf{x}$ & $\mathbf{x}$ & $\mathbf{x}$ \\
\hline Morphin unit biochemistry & $x$ & $\mathbf{x}$ & $\mathbf{x}$ & $\mathbf{x}$ & $\mathbf{x}$ & $\mathbf{x}$ \\
\hline Denzitometry $\mathrm{T}$ score $\leq-2,5$ & $\mathbf{x}$ & & & & $\mathbf{x}$ & \\
\hline Biochemistry & $x$ & $\mathbf{x}$ & $\mathbf{x}$ & $\mathbf{x}$ & $\mathbf{x}$ & $\mathbf{x}$ \\
\hline SRE detection & $x$ & $\mathbf{x}$ & $\mathbf{x}$ & $\mathbf{x}$ & $\mathbf{x}$ & $\mathbf{x}$ \\
\hline $\mathrm{AE}$ & $\mathbf{x}$ & $\mathbf{x}$ & $\mathbf{x}$ & $\mathbf{x}$ & $\mathbf{x}$ & $\mathbf{x}$ \\
\hline Scintigraphy & $\mathbf{x}$ & & $\mathbf{x}$ & & $\mathbf{x}$ & \\
\hline
\end{tabular}

Table 1: Monitoring Flow Sheet.

Doxorubicin $60 \mathrm{mg} / \mathrm{m}^{2}$ and cyclophosphamide $600 \mathrm{mg} / \mathrm{m}^{2}$ interval 21 days, AT: doxorubicin $50 \mathrm{mg} / \mathrm{m}^{2}$ and docetaxel $75 \mathrm{mg} /$ $\mathrm{m}^{2}$ int 21 days, CBDCA/GEM Carboplatin AUC 5 and gemcitabine $800 \mathrm{mg} / \mathrm{m}^{2}$ day $1+8$ int 21 days, CMF cyclophosphamide $600 \mathrm{mg} /$ $\mathrm{m}^{2}$ and fluorouracil $600 \mathrm{mg} / \mathrm{m}^{2}$ and methotrexate $40 \mathrm{mg} / \mathrm{m}^{2}$ day 1 into 21 days. Pain management was carried out in accordance with a subjective evaluation of pain, with the help of the VAS visual scale, based on the WHO scales; however, the main measurable variable was the consumption of analgesics, converted to morphine units. Treatment was initiated mostly by non-steroidal analgesics (NSE) mostly paracetamol+codeine or tramadol as tolerated, then continued with opiates in a combination of short-term ones for breakthrough pain, and long-term ones usually administered in the form of a patch. In total, opioid treatment was administered to 31 patients, i.e. 59.6\%. Radiotherapy with analgesic intention was carried out immediately after the start of monitoring in six patients, and orthopedicsurgery was carried out in five patients (spinal stabilization twice, pathological fracture of the femoral neck four times).

\section{The Results}

\section{The following conditions were considered to be SRE}

Fractures, necessity of radiotherapy, transverse spinal cord lesions, hypercalcemia. Female patients were evaluated in the course of fifteen months during the study. In 8 patients it was necessary to reduce the dosage of bisphosphonates by $50 \%$ due to light progression ( $5 x$ Zometa and $4 \mathrm{x}$ ibandronate), and the established therapy continued even with the progression. No statistically significant difference was found between the two biophosphates during the time before SRE occurred. According to the chi-square log rank test, the significance level was 0.4007 , and according to the Wilcoxon test the $\mathrm{p}$-value was 0.4904 . Clear prognostic factors for patients overall survival (OS) include, in addition to the stage of the disease, the immunohistochemical status and tumor grading, which explains the statistically significant difference between patients treated by chemotherapy or hormonal therapy. HR was 8.777 at $95 \%$ confidence limits (1.056-72.931). In patients without hormonal treatment, the risk of SRE is 8.8 times higher. The difference in OS is shown in Chart 1.

The age of patients is related to the metabolic state of the bones, to the occurrence of osteopenia, osteoporosis and pelvis fractures. In the monitored period of fifteen months, no differences in OS were found based on the age.

\section{Evaluation of the group after termination of treatment after twelve months}

$17.3 \%$ of patients died, which means that $82.7 \%$ of patients were still alive, out of which $46.2 \%$ had no fracture. Patients with the occurrence of SRE accounted for $53.5 \%$, of which $7.1 \%$ of patients had a transverse 
Citation: Brančíková D, Mechl Z, Adam Z, Protivánková M, Ostřižková L, et al. (2014) Bone Markers in the Treatment of Cancer Related Bone Disease in Patients with Metastatic Breast Cancer. J Cancer Sci Ther 6: 027-031. doi:10.4172/1948-5956.1000244

spinal lesion (2 patients). In both cases it was the primo-manifestation of the disease which was already present at the beginning of the study. Furthermore, a fracture was observed in seven patients (twice in the lower arm of the pubic bone, once in the femur coli, once in the forearm and three times in a vertebra without dislocation); in all cases, radiotherapy was administered. Only radiotherapy was administered against pain in six other patients (without a fracture). Orthopedic surgery was performed twice during the treatment and six times upon admission. During the treatment, death occurred in $17.3 \%$ of patients, of which one was diagnosed (post mortem) with double malignancy (a malignant melanoma of the right retina with dissemination to CNS); $3.6 \%$ of patients were diagnosed with ischemic stroke (despite early diagnosis without thrombolysis due to the disseminated malignancy) and $10.7 \%$ of patients died of the progression of the disease. Pulmonary embolism was observed in $25 \%$ of cases.

In this group, $25 \%$ of patients had to have their doses of analgesics increased by 1-2 morphine units per day. The number of patients with SRE reached $25 \%$; in $21 \%$ of cases a palliative radiotherapy regimen was implemented (10 x $3 \mathrm{~Gy}) ; 2$ patients were diagnosed with transverse spinal cord lesion (7.1\%). Samples drawn from both groups of patients (with and without fractures) were compared at each stage. The first samples were drawn before the therapy was initiated, and then the others at each stage. The development of marker level kinetics was also compared between the two groups. According to the Cox analysis, the significance level of p-value was 0.0452 and HR was 8.516 (95\% CI 1.047 to 69.262 ). Therefore, the recorded difference is not statistically significant, although the graphic representation shows a difference. The comparison of the first CTX sampling with overall survival was found to be significant. The patients whose CTX value in the first sampling was less than 0.425 ran an 8.5 times higher risk of death. Both groups of P1NP were evaluated after the first sampling, prior to the initiation of therapy. According to the Cox analysis, the significance level of the p-value was 0.0433 and HR was 8.673 (95\% CI 1.067 to 70.520 ). Therefore, the recorded difference was not statistically significant, although the graphic representation shows a difference. However, it is lower than CTX. The comparison of the first P1NP samples with overall survival was found to be significant. The patients whose P1NP value in the first sampling was higher than 74 run 8.7 times higher risk of death. However, the difference (the p-value is 0.6131 ) does not reach statistical significance. None of the monitored parameters can be sufficiently discriminating for clinical practice to divide patients with SRE from those without it. With the minimum required specificity of $80 \%$, the sensitivity reached was insufficient (Charts 2-5).

\section{Discussion}

This study confirms that hormone treatment is a significant predictor for the occurrence of SRE. Patients without hormonal therapy have 8.8 times higher risk of SRE, which probably results partly from the nature of cancer and also indicates the negative impact of chemotherapy-induced bone loss, while the role of age has not proved significant. The correlation of densitometrically-established osteoporosis and osteopenia according to the T-Score and bone markers was not statistically significant for the occurrence of SRE, which would correspond to an effective treatment of osteoporosis (both postmenopausal and treatment-induced) by parenteral bisphosphonates in effective doses. Therefore, the occurrence of SRE can be attributed to the bone cancer and not to osteoporosis. According to the data from literature, SRA usually occurs in $36-49 \%$ of patients treated for MBC with bone metastases during the first year of treatment; it usually takes 12 months before the first SRE occurs. In this study, there were $39.02 \%$ of SRE within 12 months. The patients whose

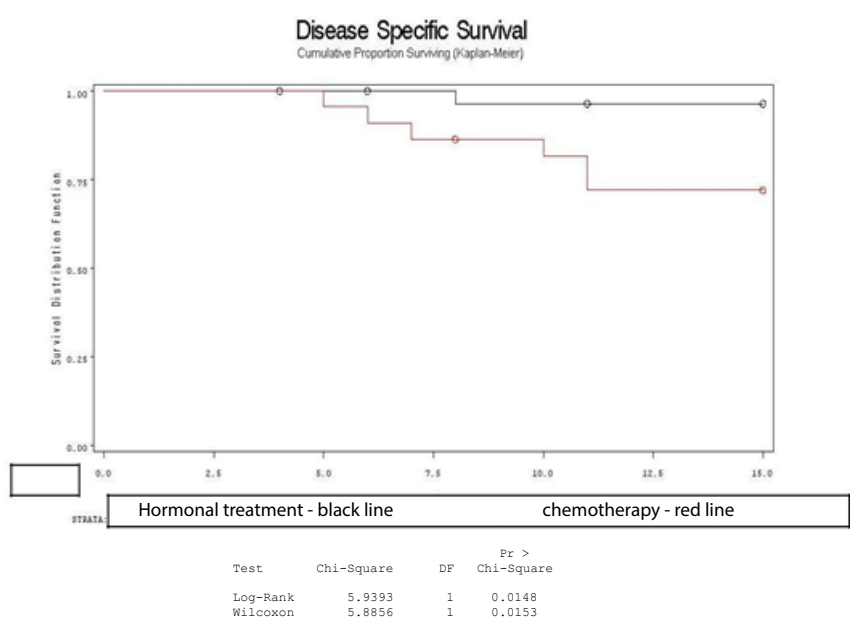

Chart 1: Disease Specific Survival [Cumulative Proportion Surviving (KaplanMeier)].

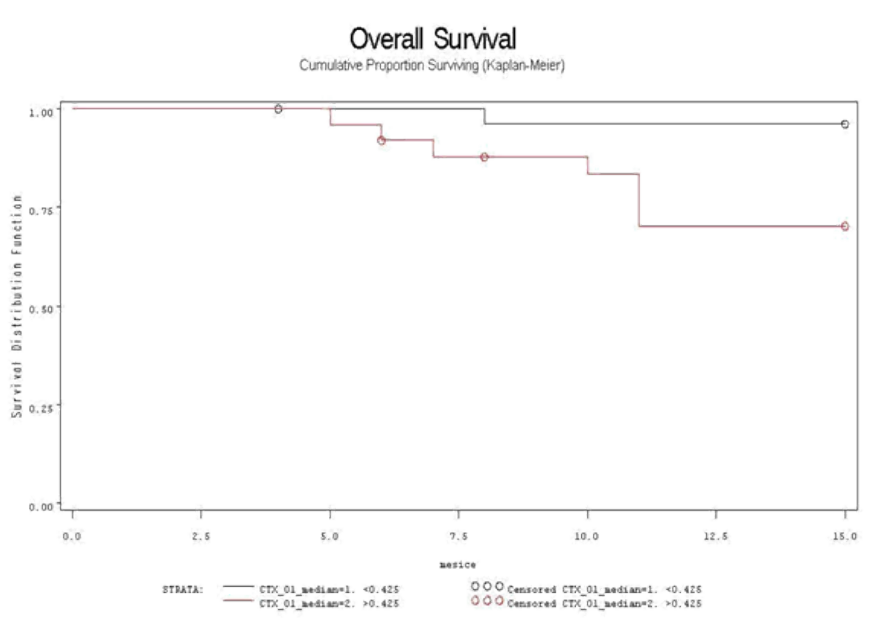

Chart 2: Overall survival according to the first CTX samples.

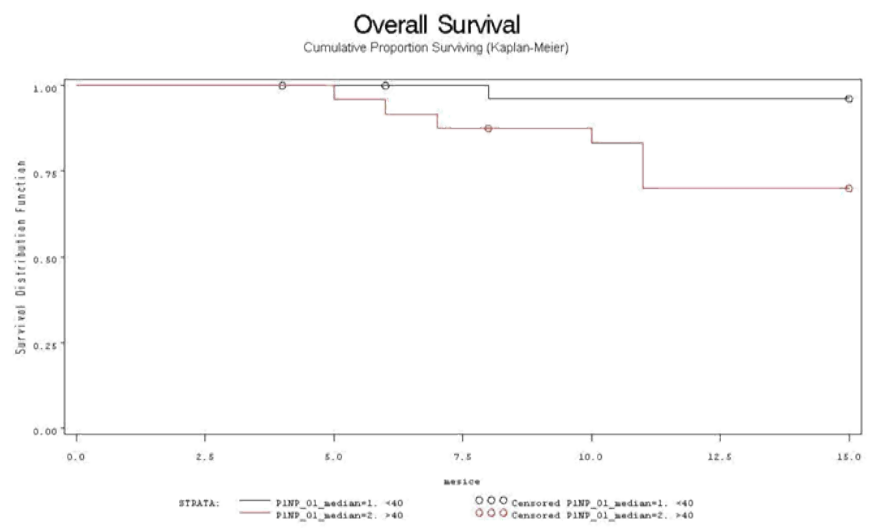

Chart 3: Overall survival according to the first P1NP samples. 
Citation: Brančíková D, Mechl Z, Adam Z, Protivánková M, Ostřižková L, et al. (2014) Bone Markers in the Treatment of Cancer Related Bone Disease in Patients with Metastatic Breast Cancer. J Cancer Sci Ther 6: 027-031. doi:10.4172/1948-5956.1000244

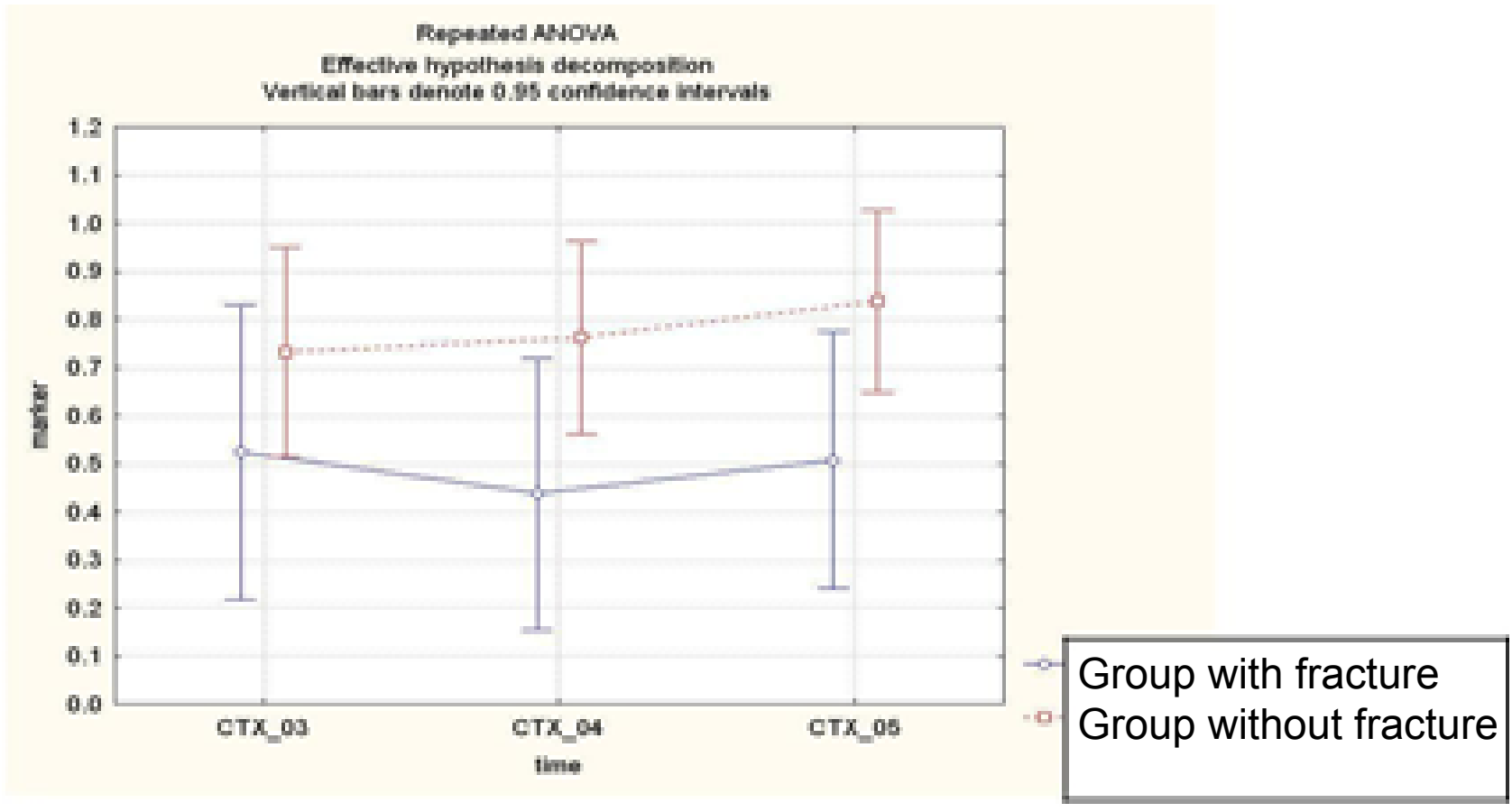

Chart 4: Comparing the kinetics of CTX levels during the therapy.

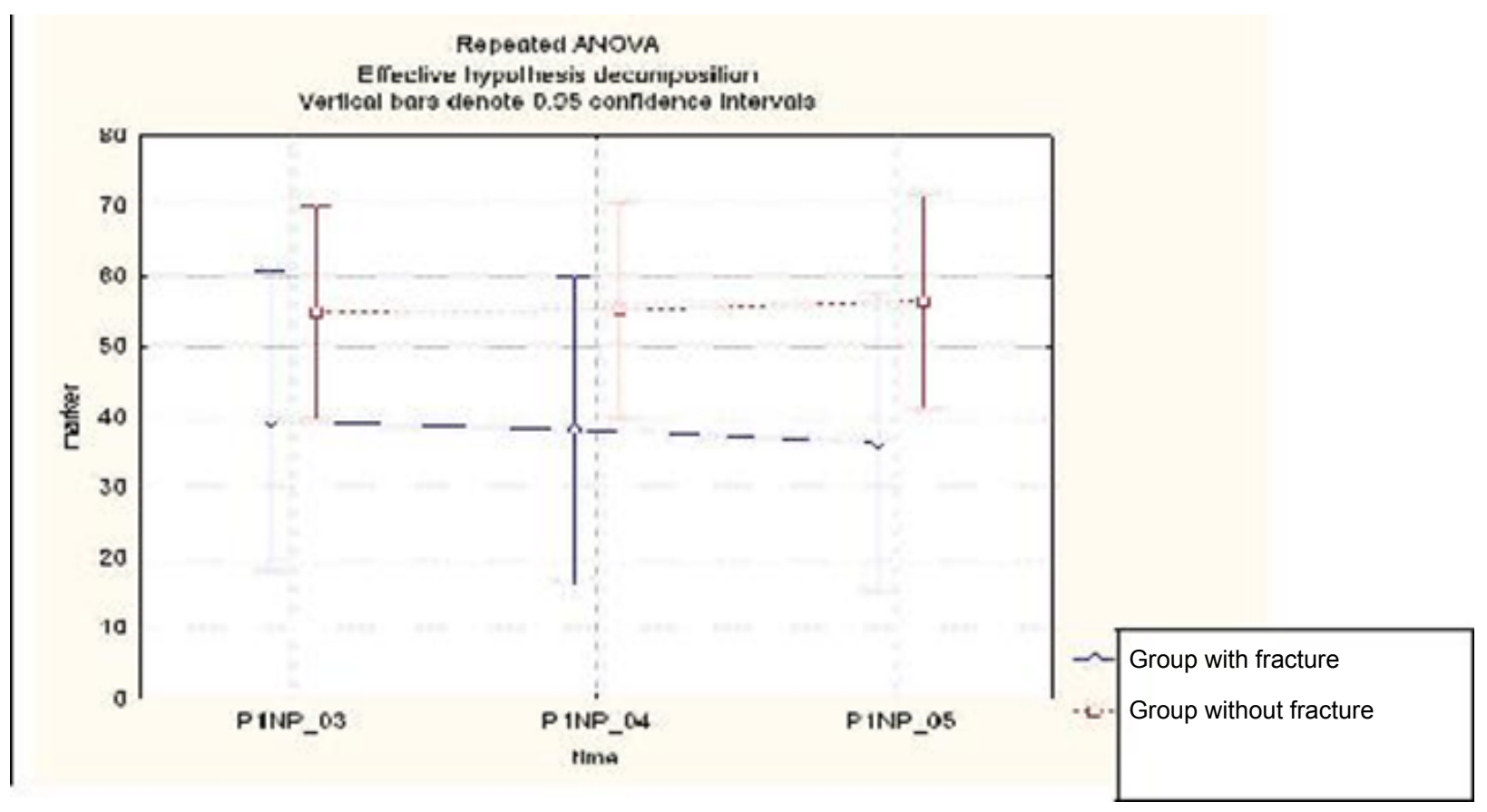

Chart 5: Comparing the kinetics of P1NP levels in patients with or without a fracture.

CTX value in the first sampling was less than 0.425 ran an 8.5 times higher risk of death; the patients whose P1NP value reached more than 74 in the first collection ran an 8.7 times higher risk of death. There is no clear explanation for this result; however, there has already been described a correlation between the higher values of P1NP in early breast cancer and the development of bone metastases and worse chances of survival [11], and in the TUGAMO study even a correlation between levels of serum CTX and P1NP in bone metastases of renal carcinoma [12]. 
Citation: Brančíková D, Mechl Z, Adam Z, Protivánková M, Ostřižková L, et al. (2014) Bone Markers in the Treatment of Cancer Related Bone Disease in Patients with Metastatic Breast Cancer. J Cancer Sci Ther 6: 027-031. doi:10.4172/1948-5956.1000244

\section{Conclusion}

The study failed to prove that testing fluctuating levels of bone markers CTX and P1NP is sufficiently specific and sensitive for predicting the occurrence of SRE in the first fifteen months of therapy. The patients whose CTX value in the first sampling was less than 0.425 ran an 8.5 times higher risk of death, and the patients whose P1NP value reached more than 74 in the first collection ran an 8.7 times higher risk of death. Therefore the value of the first sample was a negative predicative factor for survival. It cannot be confirmed that the levels of markers of bone resorption and remodeling actually copied the effect of treatment on the bone and that they were predictors of the risk of SRE, in correlation with the kinetics of the levels. The issue of the clinical significance of bone markers and selecting the most suitable ones is still under clinical testing and it cannot yet be recommended for current clinical practice when monitoring tumor bone disease.

\section{References}

1. Sassi M, Jukkola A, Riekki R, Höyhtyä M, Risteli L, et al. (2001) Type I collagen turnover and cross-linking are increased in irradiated skin of breast cancer patients. Radiother Oncol 58: 317-323.

2. De la Piedra C, Castro-Errecaborde NA, Traba ML, Méndez-Dávila C, GarcíaMoreno C, et al. (2003) Bone remodeling markers in the detection of bone metastases in prostate cancer. ClinChimActa 331: 45-53.
3. Lipton A, Costa L, Ali S, Demers L (2001) Use of markers of bone turnover for monitoring bone metastases and the response to therapy. Semin Oncol 28: 54-59.

4. Wada N, Fujisaki M, Ishii S (2001) Evaluation of bone metabolic markers in breast cancer with bone metastasis. Breast Cancer 8b: 131-137.

5. Koizumi M, Takahasi S, Ogata E (2003) Comparison of serum bone resorption markers in the diagnosis of skeletal metastasis. Anticancer Res 23: 4095-4099.

6. Schoenberg J, Rozenboom S, Wirthgen-Beyer E (2004) Evaluation of the clinical value of bone metabolic parameters for the screening of osseous metastases compared to bone scintigraphy. BMC Nuclear Medicine.

7. Yoshida K, Sumi S, Arai K (1997) Serum concentration of type I collagen metabolism as a quantitative marker of bone metastases in patients with prostate cancer. Cancer 80: 1760-1767.

8. Jensen BV, Johansen JS, Skovsgaard T, Brandt J, Teisner B (2002) Extracellula matrix building marked by the $\mathrm{N}$-terminal propeptide of procollagen type I reflect aggressiveness of recurrent breast cancer. Int J Cancer 98: 582-589.

9. BäuerleT (2008) Bevacizumab inhibits breast cancer-induced osteolysis surrounding soft tissue metastasis, and angiogenesis in rats as visualized by VCT and MRI. Neoplasia 10: 511-520.

10. Niikura N (2011) Oncologist 16: 155-164.

11. Mechl Z (2011) Současný stav a perspektivy terapie cílené na kost. Farmakoterapie7: 575-588.

12. Brančíková D (1996) Prognostic Significance of bone markers during the treatment of bone metastazes. Abstract book 19th International Congress of Anticancer Treatment. 